\title{
Performance Evaluation of Material Flow Systems in Discrete Time
}

\author{
DIPL.-WI.-ING. MARC SCHLEYER \\ PROF. DR.-ING. KAI FURMANS \\ INSTITUT FÜR FÖRDERTECHNIK UND LOGISTIKSYSTEME (IFL) \\ UNIVERSITÄT KARLSRUHE (TH)
}

Zusammenfassung

Im folgenden Beitrag werden zeitdiskrete analytische Methoden vorgestellt, mithilfe derer Informationsund Materialflüsse in logistischen Systemen analysiert und bewertet werden können. Bestehende zeitdiskrete Verfahren sind jedoch auf die Bearbeitung und Weitergabe in immer gleichen Mengen („One Piece Flow") beschränkt. Vor allem in Materialflusssystemen kommt es, bedingt durch die Zusammenfassung von Aufträgen, durch Transporte und durch Sortiervorgänge, zur Bildung von Batches. Daher wurden analytische Methoden entwickelt, die es ermöglichen, verschiedene Sammelprozesse, Batchankünfte an Ressourcen, Batchbearbeitung und Sortieren von Batches analytisch abzubilden und Leistungskenngrößen zu deren Bewertung zu bestimmen. Die im Rahmen der Entwicklungsarbeiten entstandene Software-Lösung „Logistic Analyzer“ ermöglicht eine einfache Modellierung und Analyse von praktischen Problemen. Der Beitrag schließt mit einem numerischen Beispiel.

\begin{abstract}
The purpose of this contribution is to introduce a framework of model elements and associated computation methods in order to evaluate information flow and physical processes in material flow systems. We extend available discrete time queueing models by new models for batch processes, which are very common in material flow systems. The proposed models for the analysis of batch processes are: Batch building under the capacity rule, batch building under the timeout rule, batch building under the minimum batch size rule, G/G/1-queue with batch arrivals, G/G[K,K]/1-queue, G/G[L,K]/1-queue, and batch split. In the context of our research we provide a Software-Solution called the "Logistic Analyzer" which is well-suited for the application to practical problems. Advantages and disadvantages of the proposed solution compared to simulation are discussed. The paper concludes with a numerical case.
\end{abstract}

\section{Introduction: Stochastic Modeling of Material Flow Systems}

Material flow systems can be modeled and evaluated by either simulation or analytical methods. It is crucial for the modeled real world application that stochastic events as demand, processing times, machine failures, scrap etc. are considered in an appropriate manner. Thus, queueing theory is the most suitable analytical tool in the literature for stochastic modeling of material flow systems [Barthold06]. On this level, average performance measures can be derived if some basic parameters have been determined from past observations of the system. However, performance measures based on system averages are not sufficient to verify whether the requested shipping times can be met with an acceptable probability, which usually lies between $95 \%$ and $99 \%$, possibly depending on order types.

Therefore, for the evaluation of design alternatives in respect to their ability to reach the requested sojourn time from order entry to shipping, discrete time queueing models are proposed. Hence, we use models primarily developed for the evaluation of ATM (Asynchronous Transfer Mode) networks [Ackroyd80] [Huebner95] [Gia96] [Haslinger99]. Since there is still a lack of the existence of appropriate discrete time models for material flow processes, we are motivated to find new solutions for problems in this field. Especially, models for the description of batch processes are missing. Therefore, we have developed a variety of batch queueing models in the discrete time domain. In this paper, we will give a short review of these new models. For a practical application we developed a software solution in which material flow networks can be composed and parameterized user-friendly by means of a graphical user interface. Furthermore we will discuss the main advantages of our proposed approach. The paper concludes with a numerical case. 


\section{Queueing Analysis in Discrete Time Domain}

Since time will be treated as discrete, events are only recorded at moments which are multiples of an increment $t_{i n c}$. When several events happen at the same instant it is assumed that departures from a node are served before the arrivals are. Events are created when moving items (material or orders) change their location or their status, for instance by being served, by entering a queue, or by merging with a stream of other items. Figure 1 shows the random variables characterizing the necessary input and resulting output of a $\mathrm{G} / \mathrm{G} / 1$-queue, representing a resource within a material flow network.

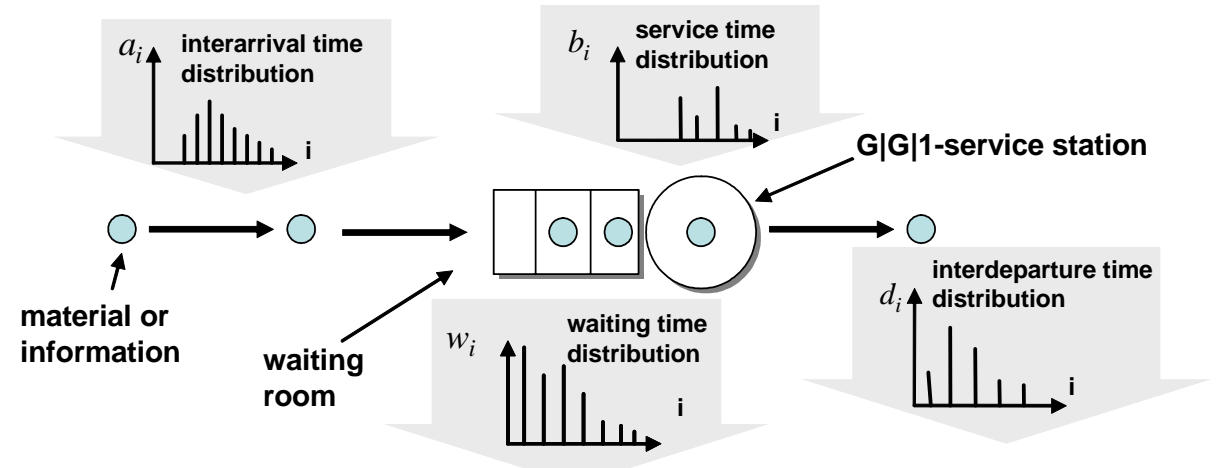

Figure 1: The G/G/1 queue in discrete time domain; the interarrival time and service time distribution as input variables, and the waiting and interdeparture time distribution as output

Subsequently, we present a short review of discrete time queueing models classified according to "one-pieceflow" and "batch flow" models.

\subsection{One-Piece Flow Modeling}

At first, we summarize briefly some basic queueing models in the discrete time domain, which allow the modeling of simple problems in material flow systems. These models are restricted to an one-piece flow.

As in the famous Queueing Network Analyzer of Whitt [Whitt83] for the analysis of general open queueing networks in the continuous time domain, we have to model the following basic operations in the discrete time domain as well:

- The service operation by means of the $\mathrm{G} / \mathrm{G} / 1-$ queue $^{1}$; the interarrival and the service time are independent and identically distributed (iid)

- The split operation in order to split an incoming stochastic stream into two or more outgoing streams

- The merge of independent stochastic streams

In Figure 2 the named basic discrete time queueing operations are depicted.
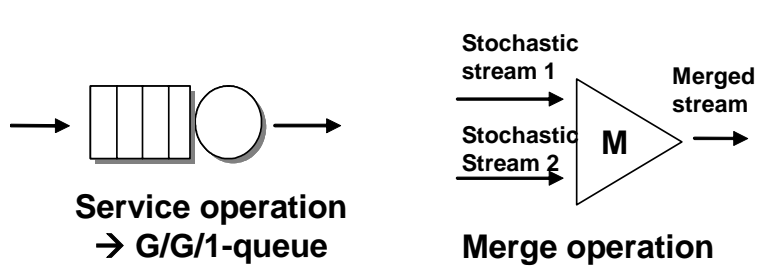

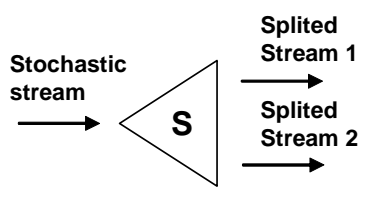

Split operation

Figure 2: Basic operations for modeling material flow networks in discrete time domain

As by Whitt it is assumed that the nodes of a material flow network are treated as stochastically independent. In order to connect the nodes, the departure process of each of the named model elements has to be known. Furthermore, for a performance evaluation the waiting times of the G/G/1-queues has to be determined. Grassman and Jain [Grassmann89] present a fast numerical method which is based on a Wiener-Hopf factorization of the underlying random walk. These authors suggest also an approach for the calculation of the interdeparture time distribution which is assumed to be the arrival stream for the succeeding node [Grassmann88].

\footnotetext{
${ }^{1}$ The Kendall's notation is widely used to classify elementary queueing systems: $\mathbf{A} / \mathbf{B} / \mathbf{m}$ - queueing discipline, where A indicates the interarrival time distribution, $B$ the service time distribution and $\mathrm{m}$ the number of servers. E.g. the letter $\mathrm{G}$ denotes generally distributed. Generally, if the queueing discipline is not indicated, it is assumed that the queueing discipline is First-Come-First-Served.
}

(C) 2007 Logistics Journal : Nicht referierte Veröffentlichungen - ISSN 1860-5923 
The analytical models for the split and merge operation computing the interdeparture times can be found in the paper of Furmans [Furmans04].

The three briefly presented basic operations allow a rough and a fast analysis of material flow systems. With the aim to increase the level of detail regarding modeling of material flow processes, we introduce subsequently new models for the description of batch processes.

\subsection{Batch Flow Modeling}

Many operations in material flow systems are done in batches. The reason for building batches is clear and evident, capacity. In most cases, it is more efficient to transport or to process a batch of entities instead of transporting or processing a single entity. Let us exemplify the necessity of building batches in more detail by presenting four basic reasons which are related to material flow systems:

- Transport/Handling: To reduce the handling effort, a specific number of material units are gathered to one transport unit. The maximum batch size is given by the capacity of the transport carrier. Examples of transport carriers are pallets, wire cages, plastic bins, etc.

- Setup: It is often necessary for industrial operations to set up machines at the beginning of a batch service. Setup operations are tool changes, cleaning operations or programming setups.

- Batch Service: There are different types of production operations in the industrial production environment, in which a certain amount of units is processed simultaneously. Examples are heating treatments in an oven or surface treatments of semiconductors in chemical washings.

- Order Pooling: For instance, the picking operation is a main task in warehouses and distribution centers. The picking time can be reduced significantly by a thoughtful grouping of the picking orders.

We propose an analytical toolkit of various mathematical models for batch processes which are: Batch building under different strategies; G/G/1-queue with batch arrivals, G/G[K,K]/1-queue, G/G[L,K]/1-queue, and the batch sorting operation. Figure 3 gives an overview of the different batch queueing models. For each model we developed mathematical approaches for the determination of the interdeparture time distribution which enables us to investigate open queueing networks where batch processes are involved. Thus, a network can be composed from a given library of stochastic model elements. Thereafter, the network can be analyzed and evaluated under various parameter configurations. The sojourn time distribution can be calculated for each stochastic model element. This gives us the probability that an order can be fulfilled in an acceptable time which is very crucial for the design of material flow systems. Depending on order types, the aspired level of order fulfillment lies usually between $95 \%$ and $99 \%$. The mean number of orders in the system can be estimated using Little's Law.

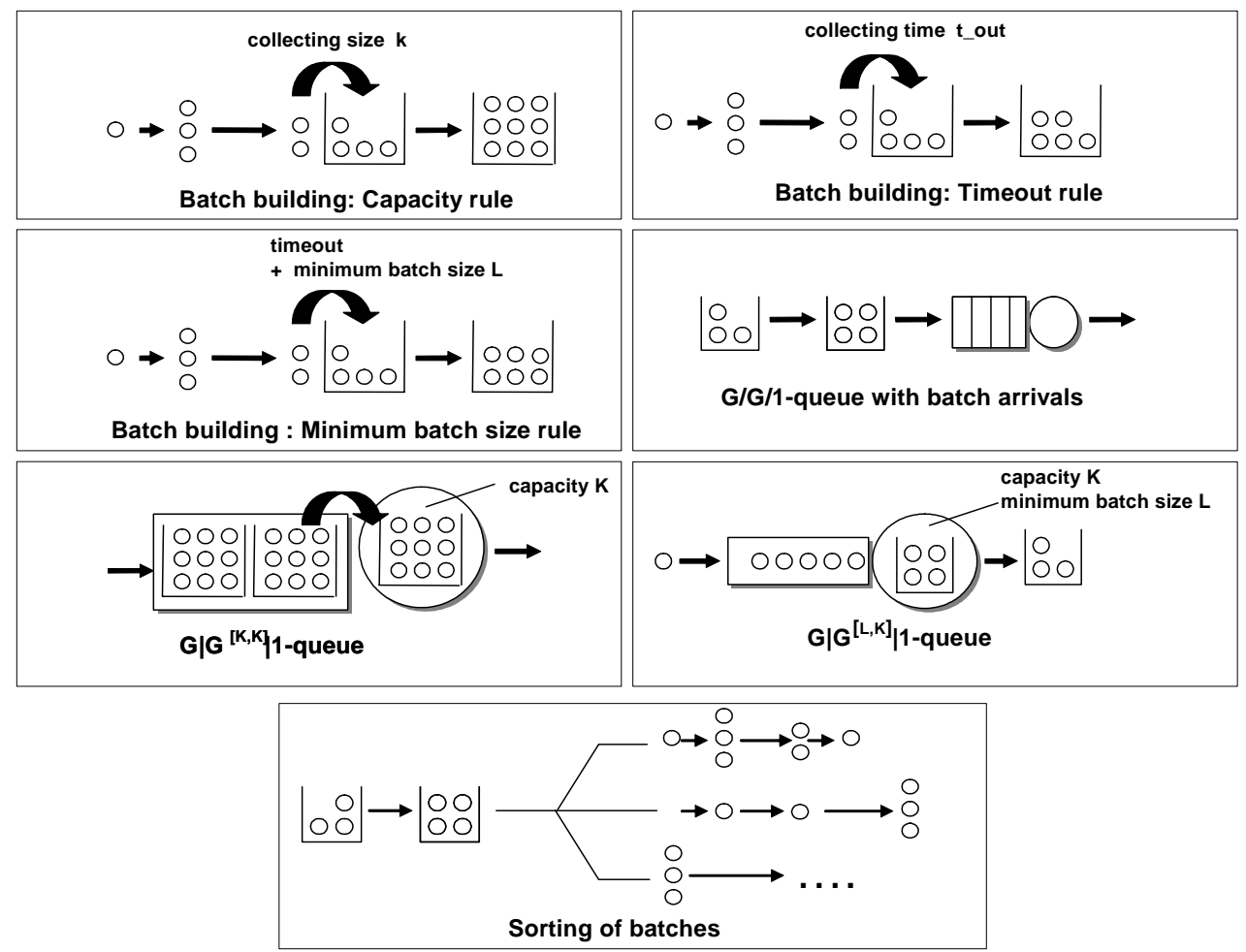

Figure 3: Discrete time models for the analysis of batch processes 
Subsequently, we present briefly the new stochastic model elements shown in Figure 3.

Batch Building: Considering material flow networks it is necessary from the economic point of view to build batches of orders. It can be distinguished between two basic batch building modes: Collecting of orders until a predetermined amount of orders is reached or until a predetermined collecting time interval is elapsed. In addition, there are possible modifications of these basic batch building modes. An example is the minimum batch size rule, in which the collecting time is at least $t_{\text {out }}$ time units. When $t_{\text {out }}$ ends and less than a minimum amount of $\mathrm{L}$ orders were collected, the batch building process continues until the required number of $\mathrm{L}$ customers is attained. For the named batch building modes we developed exact mathematical methods for the calculation of the waiting and interdeparture time distribution under general assumptions of the input distributions. The difficulty of the mathematical models is to determine the probability description of the systems state at the beginning of a batch building process. This probability description is solved using discrete homogeneous Markov chains or renewal theory. For a detailed study we refer to the paper of Schleyer and Furmans [Schleyer05].

Batch Arrival Queues: Again, Schleyer and Furmans [Schleyer06a] introduce a method for the determination of the waiting time distribution of the discrete time $\mathrm{G} / \mathrm{G} / 1$ queue with batch arrivals. It is assumed that the batch size of the arriving batch is described by an iid random variable. In addition, the distribution of number of orders at the arrival instant can be determined which can be used for the dimensioning of material flow buffers. A numerical study of the discrete time G/G/1 queue with batch arrivals shows that the quantiles of performance measures react more sensitively on parameter changes than mean values. For example, increasing unstable behavior of arrival and service processes influences the 95\%-quantile of the sojourn time distribution considerably stronger than the mean sojourn time. Therefore, focusing on mean values, the consequences of varying variability ${ }^{2}$ on the on-time order fulfillment will be underestimated.

Batch Service Queues: With the G/G[K,K]/1-queue, G/G[L,K]/1-queue we investigated in our research work two different batch service strategies, the full batch policy, where always a batch of the maximum server capacity $\mathrm{K}$ is collected and served, and the minimum batch size policy, where a service process is initiated only if at least $\mathrm{L}$ orders are accumulated in the queue [Sch06b]. In order to analyze performance measures of the $\mathrm{G} / \mathrm{G}[\mathrm{K}, \mathrm{K}] / 1$-queue, this queueing system has to be composed into two subsystems, namely a collecting station running under the capacity rule and a $\mathrm{G} / \mathrm{G} / 1$-queueing system. For the $\mathrm{G} / \mathrm{G}[\mathrm{L}, \mathrm{K}] / 1$-queue we determine the number of customers in the queue at the departure instant. Given this measure, the interdeparture and waiting time distribution can be derived. The $\mathrm{G} / \mathrm{G}[\mathrm{L}, \mathrm{K}] / 1$ can be optimized choosing an optimal $\mathrm{L}$ depending on systems costs such as operation and inventory costs.

Sorting of Batches: In contrast to the analysis of the batch building operation the sorting of batches branches a batch arrival stream in several directions for a further processing. Given the stochastic description of the batch arrival stream and the branching probability, the stochastic streams after sorting can be calculated. Sorting operations are very common in warehouse and distribution centers and in the quality control [Furmans06].

\section{Software-Solution}

Unfortunately, the application of queueing models in industry is not quite widespread even though the basic stochastic phenomenons are easily understandable and traceable.

With the aim to simplify the usage of discrete time stochastic models, we developed a software tool (see [Schleyer06c]). The user has access to a variety of algorithms via the user interface shown in Figure 4. This tool allows easy modeling, parameterizing and analyzing of material flow systems. Both one-piece flows and batch flows can be modeled. All stochastic model elements are clearly arranged in an object library, separated by elements for one-piece flows and batch flows. Arbitrary networks can be modeled via "drag and drop". Only those network elements can be connected whose departure stream is compatible to the arrival stream of the succeeding element. For example, if the departure process is a batch flow, the arrival process of the succeeding element has to be a batch flow as well.

Network parameters such as arrival and service time distributions, collecting sizes, timeout etc. can be easily entered via provided windows. Distributions can be imported from Microsoft Excel-sheets. Thus, data obtained by an as-is analysis of a material flow systems, generally available in the form of histograms, can be used directly. After a network is modeled and parameterized, it can be saved as a XML-file and uploaded for later use. The calculation results are illustrated using diagrams. In addition, all results can be exported as a Microsoft Excel-file for further analysis.

\footnotetext{
${ }^{2}$ The process variability is measured by the square coefficient of variation (scv) which is a normalized measure of statistical dispersion.
}

(C) 2007 Logistics Journal : Nicht referierte Veröffentlichungen - ISSN 1860-5923 


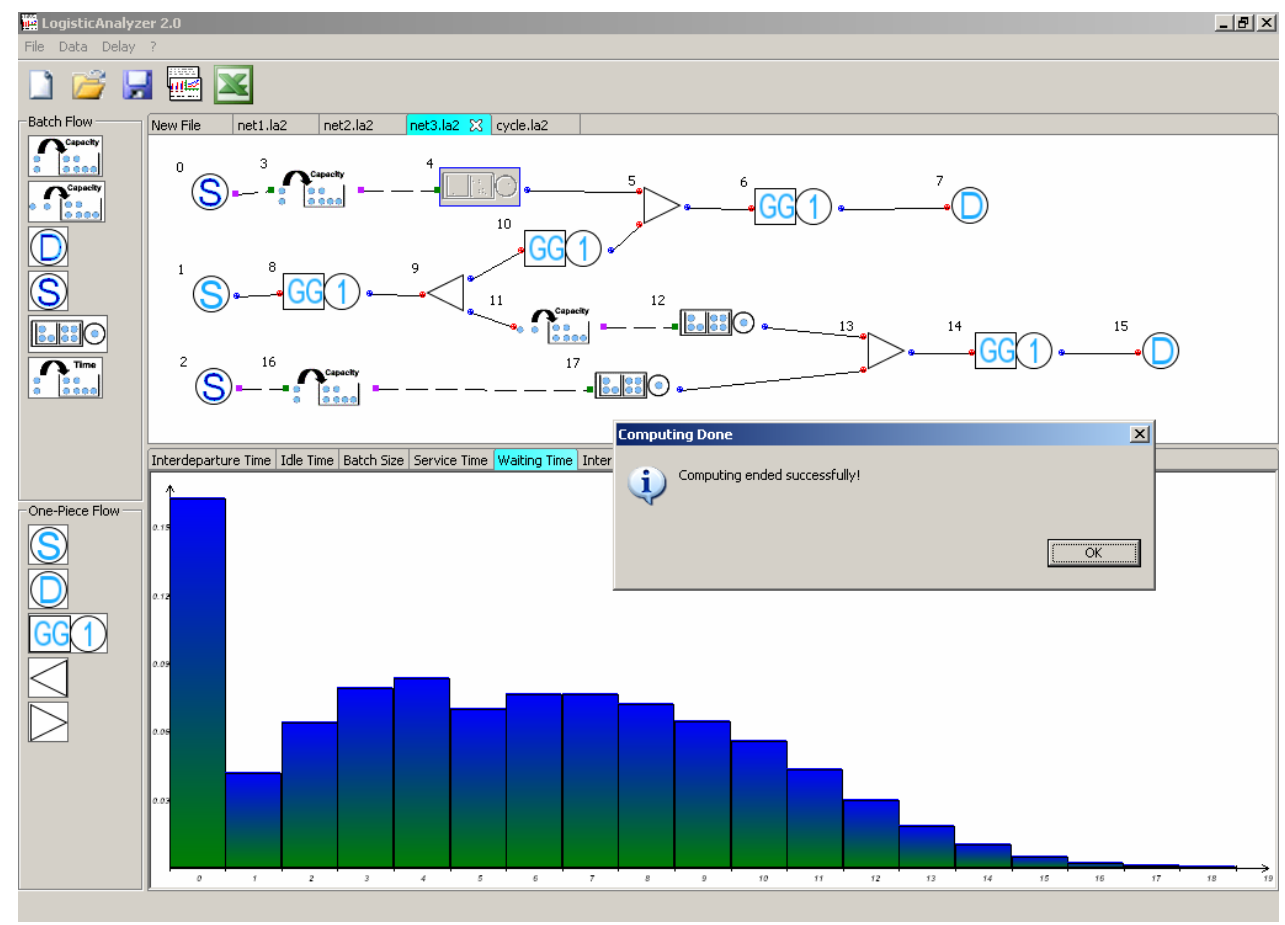

Figure 4: Software solution for a numerical analysis of material flow systems in discrete time; screenshot of the graphical user interface

The presented software is developed in the Java programming language. The user interface is developed using $\mathrm{SWT}^{3}$ due to performance reasons. We paid great attention to an easy extensibility during the development of the software solution. This is realized by a plug-in based software architecture. Thus, new algorithms can be connected to the API without any difficulty and they are immediately applicable after copying the algorithm's source code in the therefore directory.

In the next section we present a numerical case in which we used the introduced Software-Tool.

\section{Numerical Case: Order Flow Network Analysis in a Warehouse}

Let us illustrate the application of our approach by modeling an exemplary order flow in a warehouse. Furthermore, we compare the analytical results with results obtained by simulation. Figure 5 shows the order flow of our case. It is a rough process description which is often sufficient in an early planning stage. Likewise, the data available at this point is rough as well. For these reasons, an analytical tool, like the one we present here, is a practical supplement to simulation. We identify processes like receiving incoming orders, differentiated by ordinary and large goods, collecting of orders, sorting, picking, control and packaging, and outgoing orders. Given the arrival stream of the incoming orders, the service times of the underlying processes and the batch sizes for the collecting processes the sojourn time of orders in the system can be determined. Our approach enables the calculation of the probability of the on-time order fulfillment given sojourn time targets.

The order flow of Figure 5can be transferred to a queueing network using the stochastic model elements discussed previously. So, we use the elements "batch building", "sorting of batches", "G/G/1-queue with batch arrivals", "G/G/1-queue" and "stochastic merge". The resulting network of stochastic elements is illustrated in Figure 6. We identify three different order flows through the network, first from "Incoming orders 1 " to "Outgoing orders 1", second from "Incoming orders 1" to "Outgoing orders", and third from "Incoming orders 2 " to "Outgoing orders 2". Accordingly, we number them "order flow 1", “order flow 2", and "order flow 3". In Table 1 the input parameters for the network nodes are given. Thus, the throughput of the three flows are $\lambda(1)=0.427, \lambda(2)=0.284$, respectively $\lambda(3)=0.405$ orders per time unit.

\footnotetext{
${ }^{3} \mathrm{SWT}$ is an open source widget toolkit for Java designed to provide efficient, portable access to the user-interface facilities of the operating systems on which it is implemented. The SWT implementation accesses the native GUI libraries of the operating system using JNI (Java Native Interface) in a manner that is similar to those programs written using operating system-specific APIs.
} 


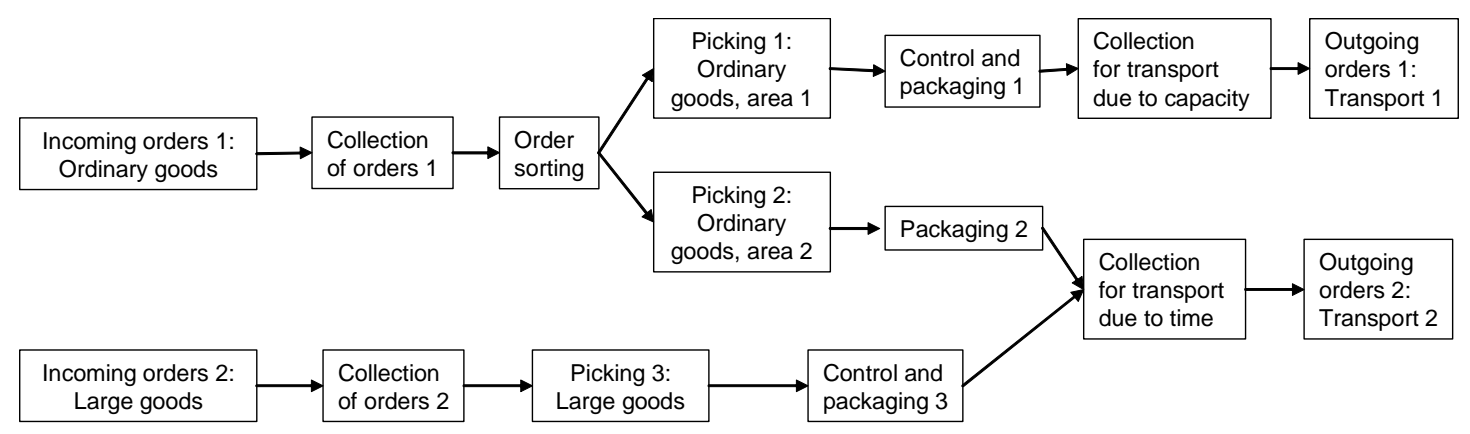

Figure 5: Example of an order flow in a warehouse

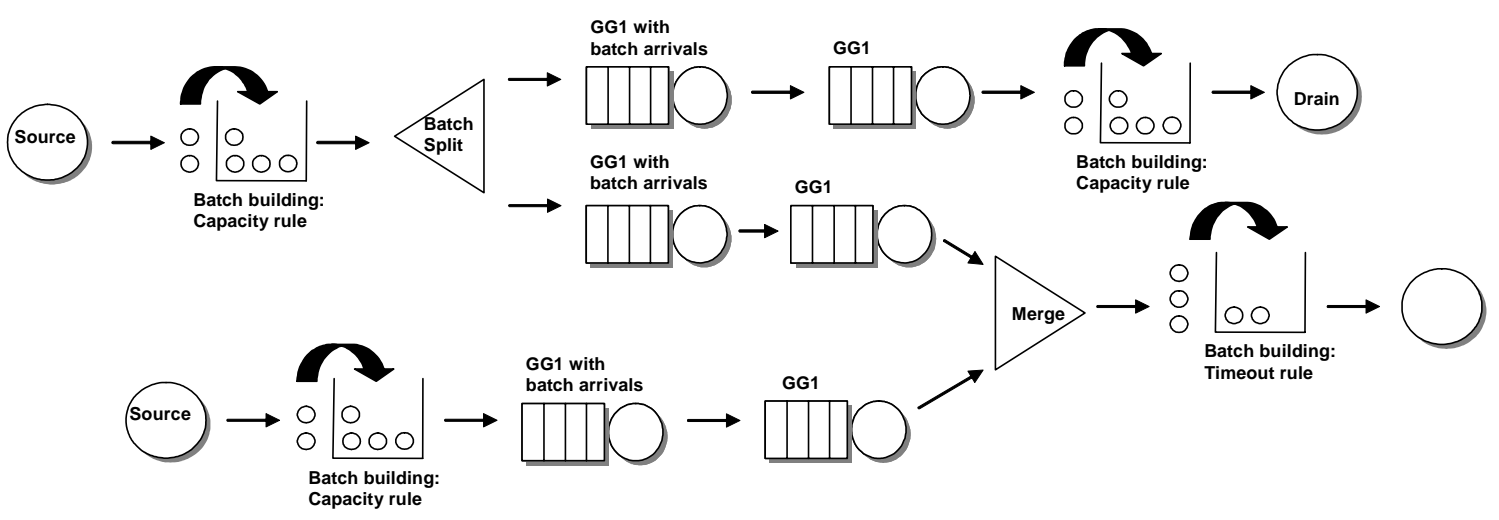

Figure 6: Network of discrete time queueing elements for an exemplary order flow in a warehouse

Modeling queueing networks we assume that the stochastic model elements are stochastically independent, which is a common assumption in queueing theory (see [Whitt83] and [Buzacott93]). It is important to mention that the departure stream of a queueing system is generally a correlated stochastic stream. Livny [Livny93] investigates this effect using simulation. Therefore we expect that there is a deviation between the analytical results and the results obtained by simulation. 
Table 1: Input variables for the numerical case

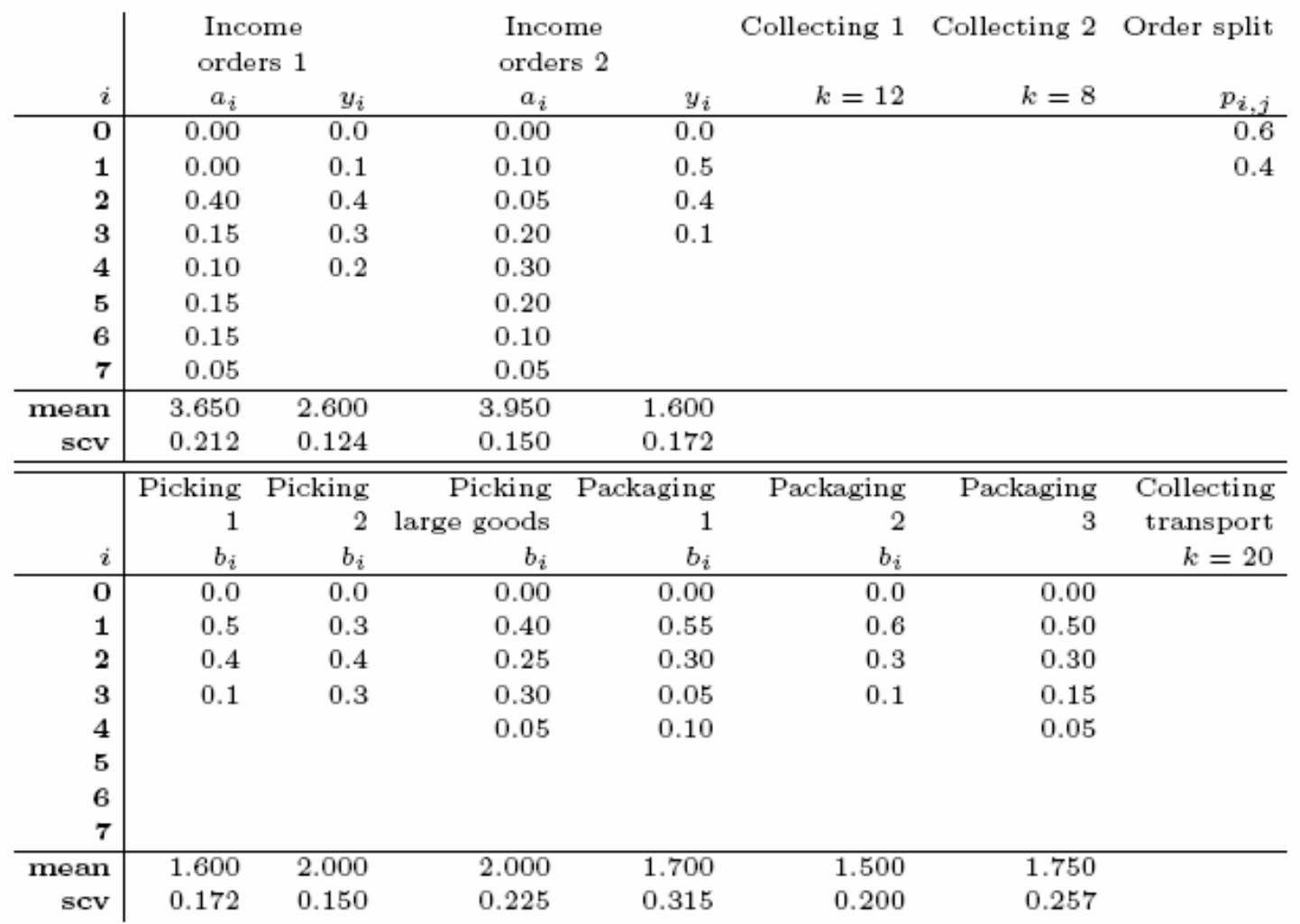

In order to test the accuracy of our analytical case we use discrete event simulation. We computed the sojourn time distribution for all three flows. The results are illustrated in Figure 7. Due to correlation effects there is a deviation between the analytical and simulation results. This deviation increases if the network becomes larger and more complex. However, the results are very promising and well suited for the analysis of the material and information flows in material handling systems. In Table 2, the mean sojourn time of orders in the network, the squared coefficient of variation of the sojourn time distribution, and the $90 \%, 95 \%$ and $99 \%$-quantile of the sojourn time distribution are given. Furthermore, the deviations from simulation results are illustrated.

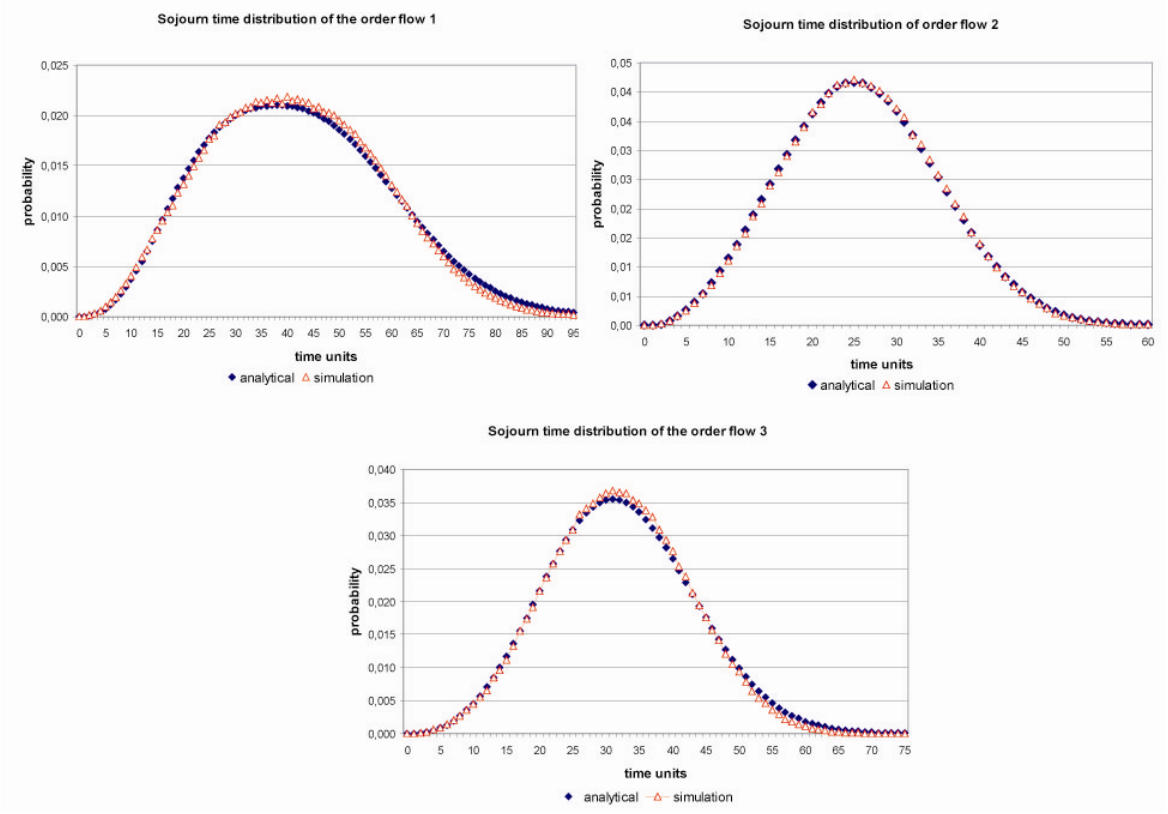

Figure 7: The sojourn time distribution of the three order flows; analytical results versus simulation 
Table 2: Numerical case results; percentage error in the sojourn time, analytical approach versus simulation

\begin{tabular}{r|rrrrrrrrrr} 
& \multicolumn{3}{|c}{ order flow 1 } & \multicolumn{3}{c}{ order flow 2} & \multicolumn{3}{c}{ order flow 3} \\
& analy & sim & \%-error & analy & sim & \%-error & analy & sim & \%-error \\
\hline mean & 42.05 & 41.46 & 1.42 & 25.86 & 25.95 & 0.32 & 32.20 & 31.89 & 0.98 \\
scv & 0.17 & 0.16 & 6.47 & 0.13 & 0.13 & 1.94 & 0.12 & 0.11 & 7.07 \\
90-quantile & 66 & 64 & 3.03 & 24 & 24 & 0.00 & 35 & 34 & 2.13 \\
95-quantile & 72 & 70 & 2.78 & 27 & 27 & 0.00 & 39 & 37 & 1.96 \\
99-quantile & 86 & 81 & 5.81 & 33 & 33 & 0.00 & 47 & 44 & 5.08
\end{tabular}

Since the computing times for the analytical methods are $l o w^{4}$, it enables an extensive "what-if" analysis for material flow systems which is necessary in the decision process at an early planning stage. This is an enormous advantage compared to simulation which is a very time consuming approach.

\section{Advantages of Modeling Material Flow Systems in Discrete Time}

If material handling systems are analyzed in discrete time using the proposed stochastic discrete time model elements, benefits related to accuracy, determination of quantiles, and efficiency are achieved.

Accuracy: An analysis of material flow systems by means of general queueing systems in a continuous time domain is based on the description of the stochastic processes by the first two moments. Concerning the G/G/1queue, the first two moments of the interarrival and service time are used to calculate the mean waiting time and the first two moments of the interdeparture time using approximations (see [Whitt83] and [Whitt93]). The accuracy of the used 2-parameter approximations is dependent on the squared coefficient of variation (scv) of the interarrival and service time distribution, and the utilization [Shanthikumar80]. In some cases the deviation from the exact solution can be remarkable. In contrast, the waiting time distribution of the discrete time G/G/1-queue estimated by the approach of Grassmann and Jain, is exact within an $\varepsilon$-neighbourhood.

We conclude that discrete time queueing analysis is an analytical tool of high accuracy.

Computation of Quantiles: The material and information flow of logistic systems should be designed in a way that guarantees the order fulfillment in a predetermined time with a chosen probability (e.g. 95\%). Hence, the distribution of the time for which an order remains in a system, defined as the sojourn time, has to be known. Then, its quantiles (e.g. the 95\%-quantile) can be determined.

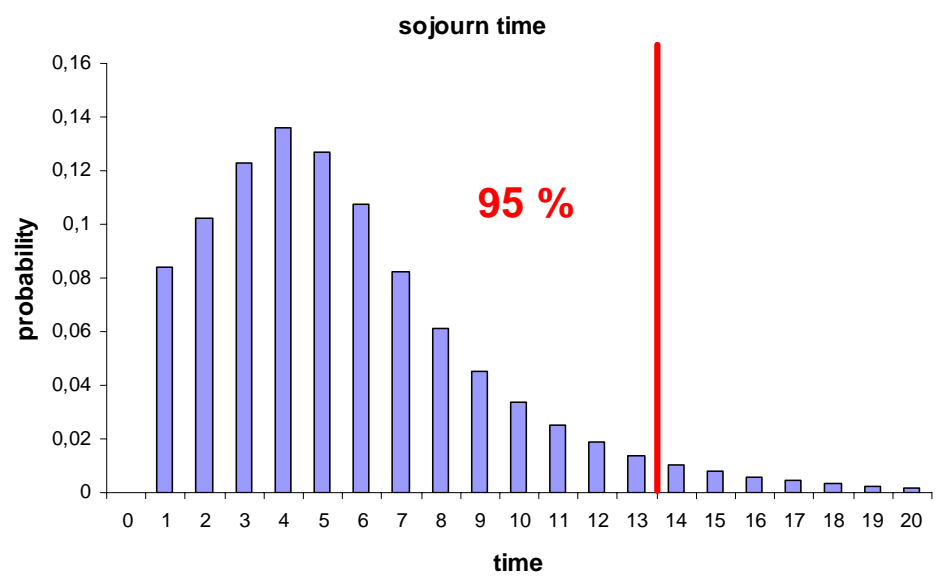

Figure 8: The 95\%-quantile of the sojourn time distribution: Design of material flow systems with a guarantee of the order fulfillment in a predetermined time with a chosen probability

Figure 8 exemplary shows the distribution of the sojourn time which describes an arbitrary material flow process. Using this distribution, we can determine the minimum time length so that the concerned process is finished with a chosen probability. This minimum time length, denoted as quantile, is 13 time units in Figure 8, and is marked by a bold line. The chosen quantile in this case is the $95 \%$-quantile. Thus, an analysis on the basis of distributions is required. This can be achieved by means of the suggested framework of stochastic discrete time elements. For all these elements, the waiting time distribution has to be determined.

\footnotetext{
${ }^{4}$ Varying milliseconds to seconds depending on the vector size of the input parameters 
Since the service time distributions are given, the sojourn time distribution can be determined and with it the probability of an on time order fulfillment.

We conclude that an analysis of material handling systems by means of discrete time queueing methods enables a more detailed description of the systems behavior than analytical methods in continuous time domain.

Processes in Real Material Flow Systems are "Discrete": The assumption that time is not continuous but discrete is essentially not a restriction for modeling material flow systems. For example, the travel time of a material handling device can only adopt a few time values, which can be very well described by a discrete distribution. Given the travel times for each possible direction and the probability choosing a direction, the service time distribution can be easily derived.

In contrast, modeling of stochastic processes in the continuous time scale requires the existence of a theoretical distribution function or the description by their moments. The derivation of the theoretical function is time consuming and this function describes the real stochastic process with imprecision. Especially, the description of multi-modal functions is difficult. On the other hand, using discrete time queueing analysis arbitrary distribution functions, in the way they exist after an as-is analysis for a material flow system, can be used. Generally, the results of an as-is analysis of a material flow system are available in the form of histograms. The normalization of these histograms yields discrete distributions functions which are the input for the analysis.

We conclude that a discrete time queueing analysis represents real processes with a high degree of accuracy, since data ascertained in an as-is analysis is generally on a discrete basis. Furthermore, low effort is required in data acquisition.

Efficiency: In industrial practice, the analysis of material flow systems is often addressed via simulation. Simulation is a very powerful tool with an enormous degree of freedom regarding modeling. Simulation models can be used for any desired level of detail. However, simulation is very time consuming and therefore expensive. It requires a lot of time for modeling, validation and performing experiments. Numerous simulation runs are required for a single experiment in order to achieve correct results within a given statistical range.

Analytical approaches are well-suited to support the long-range planning of material flow systems in an early planning stage, in which the capacities are searched to minimize facility costs under the condition of cycle time targets. In this planning stage, detailed input data ${ }^{5}$ is not available and often based on rough approximations. Therefore, a rough and extensive "what-if" analysis is required (see Hopp02]). For this kind of analysis, analytical models are well suited since they require considerably less time for conduction the numerical experiments.

We conclude that analytical methods are much more time efficient than simulation.

\section{Conclusion and Outlook}

The purpose of this paper was to give a review of new stochastic models in discrete time domain in order to analyze material flow systems. In our research, we focus on the determination of the probability of an on-time order fulfillment given time targets, since this is of vital importance for the design of material flow systems. Various models for the description of batch processes missing so far in the literature are now available for such a detailed analytical analysis. For a study of the mathematical approaches we refer to [Furmans04] [Schleyer05] [Furmans06] [Schleyer06b] and [Schleyer06c]. A Software-Solution well-suited for the application to practical problems was presented.

Finally, our approach enables to set up optimization models. Given a network of stochastic discrete time model elements for predicting the sojourn time for a given process configuration and product volume profile, it is possible to find a minimum cost configuration that satisfies given performance specifications. However, the introduction of detailed optimization models for material flow systems where batch processes are involved have to be left open for future research.

\footnotetext{
${ }^{5}$ Input data such as demand, processing times, quality rates, failure rates etc. 


\section{Literature}

[Ackroyd80]

[Barthold06]

[Buzacott93]

[Gia96]

[Grassmann88]

[Grassmann89]

[Hopp02]

[Furmans04]

[Furmans06]

[Haslinger99]

[Huebner95]

[Livny93]

[Schleyer05]

[Schleyer06a]

[Schleyer06b]

[Schleyer06c]

[Shanthikumar80]

[Whitt83]

[Whitt93]
Ackroyd, H.:Computing the waiting time distribution for the $\mathrm{G} / \mathrm{G} / 1$ queue by signal processing methods. IEEE Transactions on Communications, 38:52-58. 1980

Barthold J. and Hackman T.: Warehouse \& Distribution Science. Internet: www.warehouse-science.com. 2006

Buzacott J. and Shanthikumar G.: Stochastic models of manufacturing systems. Prentice Hall, Englewood Cliffs, NJ. 1993

Tran-Gia,P.: Analytische Leistungsbewertung verteilter Systeme. Springer. 1996 Grassmann, W. K. and Jain, J. L.: Numerical solution for the departure process from the GI/G/1 queue. Comput. Opns. Res., 15: 293-296. 1988

Grassmann, W. K. and Jain, J. L.: Numerical solutions of the waiting time distribution and idle time distribution of the arithmetic GI/G/1 queue. Operations Research, 37: 141-150. 1989

Hopp, W. J.; Spearman, M.; Chayet S., Donohue K. L., and Gel E.. Using an optimized queueing network model to support wafer fab design. IIE Transactions, 34(119-130), 2002.

Furmans K.: A framework of stochastic finite elements for models of material handling systems. In Progress in Material Handling Research: 8. International Material Handling Research Colloquium, Graz. 2004

Furmans K. and Schleyer M: An extension of the framework of stochastic finite elements for models of material handling systems. In Progress in Material Handling Research: 9. International Material Handling Research Colloquium, Salt Lake City. 2006

Hasslinger G. and Klein T: Breitband-ISDN und ATM-Netze. Teubner. 1999

Hübner F. and Tran-Gia,P.: Discrete-time analysis of cell spacing in ATM systems. Telecommunications Systems, 3: 379-395. 1995

Livny, M. and Melamed, B. and Tsiolis, A.: The impact of autocorrelation on queuing systems. Management Science, 39: 322-339.1993

Schleyer M. and Furmans K.: Analysis of batch building processes .In: Proceedings of the 20th IAR Annual Meeting, Mulhouse. P. 145-154. 2005

Schleyer M. and Furmans K.: Analytic method for calculating the waiting time distribution of a discrete time $\mathrm{G} / \mathrm{G} / 1$-queueing system with batch arrivals. OR Spectrum (online first). 2006

Schleyer M. and Furmans K.: System analysis of the discrete time G/G/1-queue with batch arrivals. In: Proceedings of the 21th IAR Annual Meeting, Nancy. 2005 Furmans K and Schleyer M.: Leistungsbewertung logistischer Systeme mit zeitdiskretem Logistikbaukasten. In: Simulation in Produktion und Logistik 2006, Tagungsband zur 12. ASIM-Fachtagung, Hrsg. Sigrid Wenzel

Shanthikumar G. and Buzacott J.: On the approximations to the single server queue. International Journal of Production Research, 18: 761-773.1980.

Whitt W.: Queueing network analyser. The Bell System Technical Journal, 62: 27792815. 1983

Whitt W.: Approximations for the GI/G/m queue. Production and Operations Management, 2: 141-161. 1993 